\title{
Recent Advances in the Design and Implementation of Practical Fiber Optical Parametric Amplifiers
}

\author{
K. K. Y. Wong*, M. E. Marhic ${ }^{\dagger}$, G. Kalogerakis ${ }^{\dagger}$, and L. G. Kazovsky ${ }^{\dagger}$ \\ *Department of Electrical \& Electronic Engineering \\ The University of Hong Kong, Pokfulam, Hong Kong \\ Email: kywong@eee.hku.hk \\ ${ }^{\dagger}$ Department of Electrical Engineering, Stanford University, Stanford, CA 94305, USA \\ Telephone: (852) 2857-8483, Fax: (852) 2559-8738
}

\begin{abstract}
Fiber optical parametric amplifiers (OPAs) are based on the third-order nonlinear susceptibility of glass fibers. If two strong pumps and a weak signal are fed into a fiber, an idler is generated. Signal and idler can grow together if pump power is high enough, and phase matching occurs. In recent years, impressive performance has been demonstrated in several respects: 1) Gain in excess of $60 \mathrm{~dB}$ has been obtained; 2) fiber OPAs can exhibit a large variety of gain spectra: a gain bandwidth of $400 \mathrm{~nm}$ has been demonstrated; tunable narrowband gain regions can also be generated; 3) Noise figure of $3.7 \mathrm{~dB}$, limited by other third-order nonlinear process; 4) Polarization-insensitive operation in both one-pump and two-pump configurations; 5) The presence of the idler can be used for wavelength conversion. Also, the spectrum of the idler is inverted with respect to that of the signal; thus by placing an OPA in the middle of a fiber span one can realize mid-span spectral inversion (MSSI) which counteracts the effect of fiber dispersion and some nonlinear effects. Besides using fiber OPA in continuouswave regime as in typical systems, pulsed-pump has also been demonstrated to achieve larger bandwidth and higher peak gain by combining with optical filtering technique. Furthermore, by modulating the pump it is possible to modulate signal and/or idler at the output. This can be used to implement a variety of signal processing functions, including: fast signal switching; demultiplexing of time-division-multiplexed signals; retiming and reshaping of waveforms; optical sampling.
\end{abstract}

A number of challenges must be overcome in order for fiber OPAs to be useful in communication applications. In multi-wavelength systems, these are: four-wave mixing, cross-phase modulation; and cross-gain modulation between signals. Furthermore, the pump-to-signal relative intensity noise (RIN) transfer and frequency/phase modulation (FM/PM) to signal intensity conversion are also potential challenges for practical fiber OPAs.

Optical amplifier; parametric amplification; wavelength conversion; optical communication; optical fibers; nonlinear optics

\section{INTRODUCTION}

Fiber optical parametric amplifiers (OPAs) are based on the third-order susceptibility $\chi^{(3)}$ of the glasses making up the fiber core. While this nonlinearity is relatively weak in silica-based glasses, the small cross-sections, low loss, and large lengths available with glass fibers can lead to sizeable effects. Fiber OPAs have the following important characteristics, which make them potentially interesting for a variety of applications:

\section{A. Gain bandwidth increasing with pump power}

This provides a means for making amplifiers with a bandwidth of several hundred nanometers, by using one or two pumps. This could provide a large bandwidth increase compared to erbium-doped fiber amplifiers (EDFAs) and Raman amplifiers (RAs), which have bandwidths of tens of nanometers.

\section{B. Arbitrary center wavelength}

The gain region can be centered about any arbitrary wavelength $\lambda_{c}$. The only constraint is that the fiber must have a zero-dispersion wavelength $\lambda_{0}$ close to $\lambda_{c}$.

\section{Large gain}

It is relatively easy to obtain large gain. Continuous-wave gain of $60 \mathrm{~dB}$, and higher pulsed gains, has been demonstrated.

\section{Idler generation}

If a pump and a signal are fed into a fiber OPA, amplified signal and residual pump emerge from the output, together with a new wavelength, the idler. This can be used for wavelength conversion. If the signal is modulated, its modulation is transferred to the idler; this can be used for shifting the optical frequency of a communication signal, which is an important function for communication networks. Conversion of unmodulated waves can also be used to generate new wavelengths for a variety of other applications.

Wavelength conversion is accompanied by spectral inversion, i.e. the exchange of modulation spectrum components about the optical carrier. This can be exploited in communication systems, to combat effects such as dispersion and detrimental nonlinear phenomena.

\section{E. High-speed optical signal processing}

The fiber nonlinearity is virtually instantaneous. Hence high-speed modulation of the pump will result in modulation of signal and idler. This can be used for regeneration of signal pulses, or optical demultiplexing of TDM signals.

\section{F. High-power capability}

Single-mode optical fibers can withstand remarkably high powers: fiber lasers with over $1 \mathrm{~kW}$ of continuous-wave (CW) 
output power have recently been demonstrated. This indicates that fiber OPAs have the potential for high-power wavelength conversion, with output of tens to hundreds of Watts.

These characteristics of fiber OPAs, indicate that they could match or exceed the performance of existing devices in various applications, such as optical amplification, or high-power wavelength conversion.

Parametric amplification in optical fibers was first observed this way by Stolen in 1975 [1]. Early work with fiber OPAs was done with pulsed lasers, with peak powers of up to $1 \mathrm{~kW}$, with which it is possible to obtain high gain in just a few meters of fiber. The emphasis was on achieving wavelength conversion between two widely-spaced but fairly narrow spectral regions, whose location was adjusted tuning the pump wavelength, $\lambda_{p}$, in the vicinity of $\lambda_{0}$. This makes it possible to obtain gain regions located several hundred nanometers away from $\lambda_{0}[2]$.

In 1996 Marhic et al. showed that by tuning $\lambda_{p}$ near $\lambda_{0}$, it is also possible to obtain gain regions that are hundreds of nanometers wide, even with commonly available communication fibers, and reasonable pump powers (of the order of $1 \mathrm{~W}$ ) [3]. This indicates that fiber OPAs could play an important role as amplifiers in future optical communication systems. Spurred in part by these prospects, as well as by steady advances of the required components, the development of fiber OPAs has been intensifying in recent years.

In this paper we consider some practical issues of fiber OPAs. We also review the state of the field that would make fiber OPAs suitable for a variety of practical applications.

\section{PRACTICAL CONSIDERATIONS}

\section{A. Stimulated Brillouin scattering (SBS)}

SBS is a high-gain nonlinear process, which can reflect most of the pump power if it exceeds a fairly modest threshold. SBS needs to be suppressed in order for fiber OPAs to operate properly. Several techniques have been demonstrated for reducing or suppressing SBS. Air gaps, optical isolators, stress distributions, or temperature distributions can be effective, but are not very practical.

Currently in most CW OPA work, SBS is suppressed by making the pump spectrum much broader than the Brillouin gain bandwidth (about $50 \mathrm{MHz}$ ). Broadening of up to several $\mathrm{GHz}$. is obtained by means of phase or frequency modulation. Various modulation schemes have been used to shape the CW pump spectrum; these include: sinusoidal phase or frequency modulation; sum of several harmonic tones; pseudo-random bit sequence (PRBS) phase modulation. SBS can also be suppressed by using a pulsed pump, with a low duty cycle. For example, 1-ns pulses will have a spectral width of the order of $1 \mathrm{GHz}$. This can be an incentive for performing experiments with pulses.

\section{B. Stimulated Raman scattering (SRS)}

The parametric gain coefficient $g$ in a well-phased-matched fiber OPA is about 2-3 times larger than the corresponding Raman contribution. As a result, it can be shown that for a broadband fiber OPA, with gain bandwidth overlapping with the Raman peak(s) due to the pump(s), the Raman contribution introduces only a minor distortion of the parametric gain spectrum. This has been shown theoretically, and experimentally verified [4]. In regions where the parametric gain is weak compared to the Raman gain, the reverse situation holds, and one may consider the Raman gain alone as a first approximation. In situations where Raman and parametric gain contributions are comparable, it is necessary to keep both in the model to accurately calculate the combined gain. This can then be done as in Ref. [4]. For two-pump OPAs, Raman interaction between the pumps needs to be taken into account if it leads to significant variation in pump powers. In that case, it is no longer possible to obtain a closed-form solution to the signal and idler equations.

\section{Longitudinal fluctuations of zero-dispersion wavelength}

So far we have assumed that the fiber dispersion properties do not change along the fiber length. In reality, it is known that essentially all practical fibers exhibit random longitudinal variations of $\lambda_{0}$. For DSF, the variance is of the order of $1 \mathrm{~nm}$, and the scale length is of the order of a few hundred meters. Measurements on some HNLF samples have revealed $\lambda_{0}$ variations as large as $20 \mathrm{~nm}$ over $1.7 \mathrm{~km}$, and rate of change in excess of $1 \mathrm{~nm}$ over $100 \mathrm{~m}$ [5],[6],[7].

The impact of $\lambda_{0}(\mathrm{z})$ on a particular OPA depends a great deal on some other OPA parameters. The pump power, $P_{0}$, is particularly important, in several ways. If it is large, one only needs a short fiber to achieve a given gain; then the probability of encountering large $\lambda_{0}$ variations is minimized. In addition, the nonlinear phase shifts which enter into the phase matching are large, and dominate over the linear phase shifts which are the only ones affected by dispersion. As a result, one should be able to obtain near-theoretical performance with high-power pulses in short fibers; this has indeed been verified [4]. On the other hand, the impact of $\lambda_{0}(\mathrm{z})$ on long fiber OPAs can be substantial [8].

\section{Fiber birefringence}

So far we have assumed that all waves remain in the same linear SOP over the entire fiber length. However, practical fibers have slight departures from ideal circular geometry, such as slightly elliptical cores, or stress due to bends, which induce the presence of linear birefringence. Furthermore, the orientation of the local polarization axes, and the birefringence, $\Delta n$, vary randomly as a function of $z$. In typical fibers, $\Delta n$ has a standard deviation of the order of $10^{-6}$. This corresponds to a beat length of the order of $1 \mathrm{~m}$, which implies that any SOP, except the local eigenpolarizations, will substantially change over $1 \mathrm{~m}$. In addition, because of the randomness of the polarization axes orientation, in a long fiber any input SOP will trace a complicated path on the Poincare sphere. The analysis of OPA performance in such circumstances is rather complex [9].

\section{E. High- $\gamma$ fibers}

If everything else is the same, a large nonlinear coefficient, $\gamma$, leads to a larger gain bandwidth, and a reduced value for the product $P_{0} L$ (N.B. $L$ is the fiber length). The advent of HNLFs [10], with $\gamma=20 \mathrm{~W}^{-1} \mathrm{~km}^{-1}$, has facilitated a number of experiments, particularly demonstrations of wideband and high-gain amplifiers. When available, they are currently the preferred medium for OPA research in the C-band.

In recent years much work has been done to develop new classes of fibers, using an array of holes around the core for confining the light, instead of a uniform low-index cladding as in conventional fibers. They have a variety of names such as holey fibers, microstructured fibers, or photonic crystal fibers. By changing the size, shape and spatial distribution of the holes, in principle one can tailor most fiber properties. For fiber 
OPAs, the potential for achieving a small mode-field diameter (and hence to increase $\gamma$ ) and to tailor dispersion are particularly attractive. It is anticipated that this approach will provide holey fibers suitable for advanced fiber OPAs.

\section{F. Pumping requirements}

The pumping requirements for fiber OPAs are substantially more demanding than for other types of optical amplifiers. The difficulties are related to: (i) the nearly instantaneous nature of the basic nonlinearity, which does not provide any smoothing of intensity fluctuations; (ii) the requirement of phase matching which imposes restrictions on the pump frequency.

\section{DEMONSTRATIONS AND APPLICATIONS}

In this section we present the main results that have been obtained with fiber OPAs, in terms of verifying their basic characteristics, as well as to address particular applications. We begin with pulsed devices, for which it is easy to obtain high pump powers, suitable for obtaining large bandwidths and high gains. We then cover $\mathrm{CW}$ devices, whose performance is important for applications such as optical communications.

\section{A. Pulsed OPAs}

OPA with $400 \mathrm{~nm}$ gain bandwidth. A fiber OPA with a gain bandwidth of $400 \mathrm{~nm}$ was reported in [5]. With such a large bandwidth, it was actually difficult to find enough signal lasers at different wavelengths to perform gain measurements. For that reason, the OPA ASE spectrum was used as a substitute for the gain spectrum (as the two are closely related). We were able to eliminate the influence of EDFA ASE from the measurements by effective filtering.

The highly-nonlinear fiber (HNLF), manufactured by Furukawa Electric, had the parameters: $L=30 \mathrm{~m} ; \gamma=18 \mathrm{~W}^{-1}$ $\mathrm{km}^{-1} ; \lambda_{0}=1554 \mathrm{~nm}$; dispersion slope, $D_{\lambda}=0.031 \mathrm{ps} \mathrm{nm}^{-2} \mathrm{~km}^{-1}$, $\beta^{(4)}=-5.8 \times 10^{-56} \mathrm{~s}^{4} \mathrm{~m}^{-1}$. The pump wavelength was $\lambda_{\mathrm{p}}=$ $1555.54 \mathrm{~nm}$. Peak pump power in the HNLF was $16 \mathrm{~W}$. A fiber Bragg grating and a circulator removed EDFA ASE from the pump. We used indirect means to estimate the bandwidth, relying on the fact that the gain spectrum is symmetric in the frequency domain. In this manner we obtained a $20-\mathrm{dB}$ gain bandwidth slightly over $400 \mathrm{~nm}$. This value is in good agreement with the theoretical value that can be calculated from the experimental parameters.

Low-noise-figure fiber OPA. The NF of a pulsed fiber OPA was recently measured by a photon-counting technique [11]. Launching weak signal pulses into the OPA ensured that they were very close to being in a coherent state (CS), the standard assumption for the derivation of NF. ' 0 ' and ' 1 ' pulses were launched to model a digital communication system. The probability density functions (pdf's) for photon numbers at the OPA output were measured for both cases, and were found to be in excellent agreement with theoretical predictions. The OPA noise figure was calculated by $N F=(1+$ $2 N / \eta) / G_{\mathrm{s}}$, where $N$ denotes the measured mean number of output ASE photons for ' 0 ' pulses, and $\eta$ is the quantum efficiency of the detection system. The graph of $N F$ versus gain asymptotically approaches a value corresponding to about 3.7 $\mathrm{dB}$ for large gain. This is larger than the $3 \mathrm{~dB}$ standard quantum limit. The difference is attributed to the effect of Raman gain [12].

TDM Demultiplexing. In a TDM system, the pulses associated with a number $N_{\mathrm{c}}$ of channels are interlaced in the time domain, and grouped in frames of $N_{\mathrm{c}}$ pulses. The role of the demultiplexer is to pick out the $n^{\text {th }}$ pulse in each frame. The demultiplexing can be destructive, in the case where the pulses associated with the other channels are lost in the process. Or it can in principle be non-destructive, if all the other pulses are preserved; in this case, further stages of demultiplexing can extract the other channels.

To make a TDM demultiplexer from a pulsed-pump OPA, one needs to have only one pump pulse per frame, but its duration should be that of a signal bit. The pump pulses must be synchronized with the bits to be extracted. Then, at the OPA output the signal pulses coinciding with the pump pulses will be amplified. If the gain is large, say 20 to $30 \mathrm{~dB}$, most of the energy in a frame could correspond to the desired channel. Then one could ignore the contributions from the other channels, and use this output to extract the desired channel. On the other hand, if the gain is too low, or the frame are long, the unamplified bits could contribute a significant amount of energy per frame. Then, it might be preferable to use the idler as the output of the DMUX. The advantage of the idler is that only the desired pulses are present, which leads to a better OSNR. With the idler, one could use relatively low gain, and still obtain good OSNR. TDM demultiplexing using the signal gain has been demonstrated for $10 \mathrm{~Gb} / \mathrm{s}$ signals [13].

$2 R$ or $3 R$ regeneration. In long-haul communication systems signals may become distorted, because of the effects of fiber nonlinear effects, dispersion, and noise added by optical amplifiers. As a result, if one considers an RZ transmission system, the bits can get corrupted in a number of ways. It may thus be desirable to restore the initial pulse shapes as much as possible. This is referred to as regeneration. Many types of regenerators have been investigated; some of them correcting just one defect, while others correct all of them. Depending on the number of defects being corrected, they are called $1 R, 2 R$, or $3 \mathrm{R}$ regenerators.

Fiber OPAs can be used for making regenerators. The idea is to use a pump which is pulsed at the same rate as the data, and synchronized with it. Since the OPA gain is a strong function of instantaneous pump power, if the pump pulses have short rise and fall times, the amplified signals pulses will be strong only during the pump pulses. This will provide reshaping and retiming of the ' 1 ' pulses. This type of regeneration is desirable because the optical frequency of the pulses is not altered, which implies that such regenerators can be cascaded. This type of regeneration has been experimentally demonstrated [14].

Optical sampling. Lasers can generate short pulses at high repetition rates. These can be used for sampling in the optical domain. This requires a very fast optical device, which responds much faster than the sampling pulses. Fiber OPAs can respond on a femtosecond time scale, and therefore can be used for optical sampling. The idea is similar to that of an optical DMUX, except that now the pump consists of pulses much shorter than the bit period. There is one pump pulse for every $N_{\mathrm{p}}$ periods, occurring at a slightly different time. Then at the OPA output the idler consists of pulses coinciding with the pump pulses, with about the same duration. These pulses, with a relatively low repetition rate, can now be converted to lowfrequency electrical signals, which can be displayed on a conventional oscilloscope. The result is a display of the detailed structure of the periodic optical signal of interest, over a one-period interval. This idea has been experimentally demonstrated [15]: by using OPA pump pulses with picosecond duration, the authors were able to implement 
optical sampling, and to display a time-resolved periodic 300 $\mathrm{GHz}$ waveform.

\section{B. CW OPAs}

CW OPA with $60 \mathrm{~dB}$ gain. Advanced SBS suppression techniques must be used for obtaining high CW gains. In Ref. [16], an isolator was used in combination with pump phase modulation for SBS suppression. Phase modulation was implemented by means of two cascaded phase modulators: the first one was driven by a sine wave at $2.5 \mathrm{GHz}$, and the second one by a pseudo-random bit sequence (PRBS). The fiber was a 1-km long HNLF, with $\gamma=17 \mathrm{~W}^{-1} \mathrm{~km}^{-1}$. With the phase modulation only, it was not possible to exceed the performance of the previous OPAs. After adding the isolator, a maximum gain of $60 \mathrm{~dB}$ was measured. The gain was actually no longer limited by SBS, but by saturation of the gain by ASE

CW OPAs with low optical noise figure. While OPAs in principle have the potential for approaching the $3 \mathrm{~dB}$ quantum limit, a number of practical difficulties have to be overcome to do so. A major difficulty is EDFA ASE. If it is not reduced by optical filtering prior to combining, it is then amplified to a very high level. To avoid this, the pump EDFA ASE must be greatly reduced by filtering before combining with the signal. This idea was first implemented in Ref. [17]. By using an FBG, the EDFA ASE was reduced essentially to a level equal to that of vacuum fluctuations near $1560 \mathrm{~nm}$, the peak of the EDFA gain. NF measurements over the OPA gain bandwidth gave a fairly constant NF value, with an average of $4.2 \mathrm{~dB}$.

Polarization-insensitive OPAs. A severe difficulty for fiber OPAs is that in their simplest forms they exhibit gain which is a function of the SOP of the incident signal, which is undesirable in optical communication systems. A variety of means have been used to overcome polarization sensitivity in various devices, and variations on these have been investigated for fiber OPAs. Good results have been obtained [18], [19].

Amplitude-noise reduction. This application is based on the fact that the gain of fiber OPAs saturates as the signal input power increases beyond a level $P_{\text {sat }}$. As a result, fluctuations around the high input level result in relatively small fluctuations around the high output level. Thus, if one considers a binary input signal, with a low level near 0 , and a high level above $P_{\text {sat, }}$, the resulting binary output waveform has noise for the high level which is compressed. In other words, we have amplitude-noise reduction for the high level. This mechanism has been studied theoretically and experimentally [20], and it has been verified that it does lead to a reduction in amplitude noise. This feature has also been used in combination with timing jitter reduction [21].

\section{CONCLUSIONS}

Substantial progress has been made in recent years with the development of fiber OPAs. Important features have been demonstrated, including: high gain; gain bandwidth of several hundred nanometers; polarization-independent configurations; low noise figure; pulsed-pump operation for a variety of signal processing operations; mid-span spectral inversion for dispersion compensation in communication networks. We anticipate that further progress with high-power pumps, highlynonlinear fibers with tailored dispersion properties, and SBS suppression techniques, fiber OPAs and related devices will find practical applications in areas such as high-power wavelength conversion and optical communication.
ACKNOWLEDGMENT

This work was supported by National Science Foundation grant ANI-0123441.

\section{REFERENCES}

[1] Stolen, R. H., "Phase-matched-stimulated four-photon mixing in silicafiber waveguides," IEEE J. Quantum Electron., QE 11, pp. 100-103 (1975).

[2] Lin, C., Reed, W. A., Pearson, A. D., and Shang, H.-T., "Phase matching in the minimum-chromatic-dispersion region of single-mode fibers for stimulated four-photon mixing," Opt. Lett., 6, pp 493-495 (1981).

[3] Marhic, M.E., Kagi, N., Chiang, T.-K., and Kazovsky, L.G., "Broadband fiber optical parametric amplifiers," Optics Letters, 21, pp. 573-575 (1996).

[4] Ho, M.-C., Uesaka, K., Marhic, M., Akasaka, Y., and Kazovsky, L.G., "200-nm-bandwidth fiber optical amplifier combining parametric and Raman gain," J. Lightwave Technol., 19, pp. 977-981 (2001).

[5] Marhic, M. E., Wong, K. K. Y., and Kazovsky, L. G., "Wideband tuning of the gain spectra of one-pump fiber optical parametric amplifiers," IEEE J. of Selected Topics in Quantum Electron., in press (2004).

[6] Mussot, A., Lantz, E., Sylvestre, T., Maillotte, H., Durécu-Legrand, A., Simonneau, C., and Bayart, D., "Zero-dispersion wavelength mapping of a highly nonlinear optical fibre-based parametric amplifier," ECOC'04, paper Tu3.3.7.

[7] Ho, M.-C., PhD Dissertation, Electrical Engineering Department, Stanford University (2001).

[8] Yaman, F., Liu, Q., Radic, S., and Agrawal, G. P., "Impact of dispersion fluctuations on dual-pump fiber-optic parametric amplifiers," IEEE Photon. Technol. Lett., 16, pp. 1292-1294 (2004).

[9] Liu, Q., and Agrawal, G. P., "Effects of polarization-mode dispersion on fiber-based parametric amplification and wavelength conversion," Opt. Lett., 29, pp. 1114-1116 (2004).

[10] Holmes, M. J., Williams, D. L., and Manning, R. J., "Highly nonlinear optical fiber for all optical processing applications," IEEE Photon. Technol. Lett., 7, pp. 1045-1047 (1995).

[11] Voss, P.L., Tang, R., and Kumar, P., "Measurement of the photon statistics and the noise figure of a fiber-optic parametric amplifier," Opt Lett., 28, pp. 549-551 (2003).

[12] Voss, P. L., and Kumar, P., "Raman-noise-induced noise-figure limit for $\chi^{(3)}$ parametric amplifiers," Opt. Lett., 29, pp. 445-446 (2004).

[13] Hansryd, J., and Andrekson, P.A., "O-TDM demultiplexer with 40-dB gain based on a fiber optical parametric amplifier," IEEE Photon Technol. Lett., 13, pp. $732-734$ (2001).

[14] Su, Y., Wang, L., Agrawal, A., and Kumar, P., "Simultaneous 3R regeneration and wavelength conversion using a fiber-parametric limiting," OFC'01, paper MG4.

[15] Li, J., Hansryd, J., Hedekvist, P. O., Andrekson, P. A., and Knudsen, S. N., "300-Gb/s eye-diagram measurement by optical sampling using fiber-based parametric amplification," IEEE Photon. Technol. Lett., 13, pp. 987-989 (2001)

[16] Wong, K. K. Y., Shimizu, K., Uesaka, K., Kalogerakis, G., Marhic, M. E., and Kazovsky, L. G., "Continuous-wave fiber OPA with $60 \mathrm{~dB}$ gain using a novel two-segment design," IEEE Photon. Technol. Lett., 15, pp. 1707-1709 (2003).

[17] Blows, J. L., and French, S. E., "Low-noise-figure optical parametric amplifier with a continuous-wave frequency-modulated pump," Opt. Lett., 27, pp. 491-493 (2002).

[18] Wong, K. K. Y., Marhic, M. E., Uesaka, K., and Kazovsky, L. G., "Polarization-independent one-pump fiber-optical parametric amplifier,' IEEE Photon. Technol. Lett., 14, pp. 1506-1508 (2002).

[19] Wong, K. K. Y., Marhic, M. E., Uesaka, K., and Kazovsky, L. G., "Polarization-independent two-pump fiber optical parametric amplifier," IEEE Photon. Technol. Lett., 14, pp. 911-913 (2002).

[20] Inoue, K., and Mukai, T., "Experimental study on noise characteristics of a gain-saturated fiber optical parametric amplifier," J. Lightwave Technol., 20, pp. 969-974 (2002).

[21] Shimizu, K., Kalogerakis, G., Marhic, M. E., and Kazovsky, L. G., "Timing-jitter and amplitude-noise reduction by a chirped pulsed-pump fiber OPA", OFC'03, Atlanta, GA, March 2003, paper TuH5, pp. 197198. 